\title{
Prolymphocyte to Leukocyte Ratio
}

National Cancer Institute

\section{Source}

National Cancer Institute. Prolymphocyte to Leukocyte Ratio. NCI Thesaurus. Code C64829.

The determination of the ratio of prolymphocytes compared to leukocytes in a blood sample. The measurement may be expressed as a ratio or percentage. 